\title{
KERNELS BY MONOCHROMATIC PATHS AND COLOR-PERFECT DIGRAPHS
}

\author{
Hortensia Galeana-SÁnchez ${ }^{1}$ and Rocío SÁnchez-López \\ Instituto de Matemáticas UNAM, Ciudad Universitaria \\ Circuito Exterior 04510, México, D.F., México \\ e-mail: hgaleana@matem.unam.mx \\ usagitsukinomx@yahoo.com.mx
}

\begin{abstract}
For a digraph $D, V(D)$ and $A(D)$ will denote the sets of vertices and arcs of $D$ respectively. In an arc-colored digraph, a subset $K$ of $\mathrm{V}(D)$ is said to be kernel by monochromatic paths (mp-kernel) if (1) for any two different vertices $x, y$ in $N$ there is no monochromatic directed path between them ( $N$ is mp-independent) and (2) for each vertex $u$ in $V(D) \backslash N$ there exists $v \in N$ such that there is a monochromatic directed path from $u$ to $v$ in $D$ ( $N$ is mp-absorbent). If every arc in $D$ has a different color, then a kernel by monochromatic paths is said to be a kernel. Two associated digraphs to an arc-colored digraph are the closure and the color-class digraph $\mathscr{C}_{C}(D)$. In this paper we will approach an mp-kernel via the closure of induced subdigraphs of $D$ which have the property of having few colors in their arcs with respect to $D$. We will introduce the concept of color-perfect digraph and we are going to prove that if $D$ is an arc-colored digraph such that $D$ is a quasi color-perfect digraph and $\mathscr{C}_{C}(D)$ is not strong, then $D$ has an mp-kernel. Previous interesting results are generalized, as for example Richardson's Theorem.
\end{abstract}

Keywords: kernel, kernel perfect digraph, kernel by monochromatic paths, color-class digraph, quasi color-perfect digraph, color-perfect digraph.

2010 Mathematics Subject Classification: 05C20, 05C38, 05C69.

\section{REFERENCES}

[1] P. Arpin and V. Linek, Reachability problems in edge-colored digraphs, Discrete Math. 307 (2007) 2276-2289.

doi:10.1016/j.disc.2006.09.042

\footnotetext{
${ }^{1}$ Research supported by UNAM-DGAPA-PAPIIT IN106613-2 and CONACyT 219840.
} 
[2] J. Bang-Jensen and G. Gutin, Digraphs: Theory, Algorithms and Applications (Springer, London, 2000).

[3] C. Berge, Graphs (North-Holland, Amsterdan, 1989).

[4] C. Berge and P. Duchet, Perfect graphs and kernels, Bull. Inst. Math. Acad. Sin. 16 (1988) 263-274.

[5] C. Berge and P. Duchet, Recent problems and results about kernels in directed graphs, Discrete Math. 86 (1990) 27-31. doi:10.1016/0012-365X(90)90346-J

[6] E. Boros and V. Gurvich, Perfect graphs, kernels and cores of cooperative games, RUTCOR Research Report 12 (Rutgers University, April 2003).

[7] V. Chvátal, On the computational complexity of finding a kernel, Report CRM300, Centre de Recherches Mathématiques (Université de Montréal, 1973).

[8] P. Duchet, Graphes noyau-parfaits, Ann. Discrete Math. 9 (1980) 93-101. doi:10.1016/S0167-5060(08)70041-4

[9] A.S. Fraenkel, Planar Kernel and Grundy with $d \leq 3, d_{\text {out }} \leq 2, d_{\text {in }} \leq 2$, are NPcomplete, Discrete Appl. Math. 3 (1981) 257-262. doi:10.1016/0166-218X(81)90003-2

[10] A.S. Fraenkel, Combinatorial games: Selected bibliography with a succinct gourmet introduction, Electron J. Combin. 14 (2009) DS2.

[11] H. Galeana-Sánchez and V. Neumann-Lara, On kernels and semikernels of digraphs, Discrete Math. 48 (1984) 67-76. doi:10.1016/0012-365X(84)90131-6

[12] H. Galeana-Sánchez, On monochromatic paths and monochromatic cycles in edge coloured tournaments, Discrete Math. 156 (1996) 103-112. doi:10.1016/0012-365X(95)00036-V

[13] H. Galeana-Sánchez, Kernels in edge coloured digraphs, Discrete Math. 184 (1998) 87-99. doi:10.1016/S0012-365X(97)00162-3

[14] H. Galeana-Sánchez, Kernels by monochromatic paths and the color-class digraph, Discuss. Math. Graph Theory 31 (2011) 273-281. doi:10.7151/dmgt.1544

[15] H. Galeana-Sánchez and R. Rojas-Monroy, A counterexample to a conjecture on edge-coloured tornaments, Discrete Math. 282 (2004) 275-276. doi:10.1016/j.disc.2003.11.015

[16] S. Minggang, On monochromatic paths in m-coloured tournaments, J. Combin. Theory Ser. B 45 (1988) 108-111. doi:10.1016/0095-8956(88)90059-7

[17] J. von Neumann and O. Morgenstern, Theory of Games and Economic Behavior (Princeton University Press, Princeton, 1944). 
[18] V. Neumann-Lara, Seminúcleos de una digráfica, An. Inst. Mat. 11 (1971) 55-62.

[19] M. Richardson, Solutions of irreflexive relations, Ann. of Math. (2) 58 (1953) 573-590. doi: $10.2307 / 1969755$

[20] M. Richardson, Extensions theorems for solutions of irreflexive relations, Proc. Natl. Acad. Sci. USA 39 (1953) 649-655. doi:10.1073/pnas.39.7.649

[21] B. Sands, N. Sauer and R. Woodrow, On monochromatic paths in edge coloured digraphs, J. Combin. Theory Ser. B 33 (1982) 271-275. doi:10.1016/0095-8956(82)90047-8

Received 18 August 2014

Revised 23 June 2015

Accepted 23 June 2015 